\title{
Energetic Electron Enhancements below the Radiation Belt and X-Ray Contamination at Low-Orbiting Satellites
}

\author{
Alla V. Suvorova, ${ }^{1,2}$ Alexei V. Dmitriev, ${ }^{1,2}$ and Chien-Ming Huang ${ }^{1}$ \\ ${ }^{1}$ Institute of Space Science, National Central University, No 300 Jungda Road, Jhongli, Taoyuan 32001, Taiwan \\ ${ }^{2}$ Skobeltsyn Institute of Nuclear Physics, Lomonosov Moscow State University, Moscow 119234, Russia \\ Correspondence should be addressed to Alla V. Suvorova; suvorova_alla@yahoo.com
}

Received 29 August 2014; Accepted 21 September 2014; Published 9 October 2014

Academic Editor: Athina Meli

Copyright (c) 2014 Alla V. Suvorova et al. This is an open access article distributed under the Creative Commons Attribution License, which permits unrestricted use, distribution, and reproduction in any medium, provided the original work is properly cited.

The work concerns a problem of electron-induced contaminant at relatively low latitudes to high-energy astrophysical measurements on board the low-orbiting satellites. We show the results of a statistical analysis of the energetic electron enhancements in energy range 30-300 keV observed by a fleet of NOAA/POES low-orbiting satellites over the time period from 1999 to 2012 . We demonstrate geographical distributions of great and moderate long-lasting enhancements caused by different type of the solar wind drivers.

\section{Introduction}

Instrumental measurements in high-energy astrophysics require knowledge of contaminating background radiation of local (magnetospheric) origin [1]. Either by direct penetration or by secondary radiations produced in payload materials, photon detectors may at times give spurious responses, particularly if the "background" radiations are nonsteady [2]. It was recently pointed out that the most important effect limiting the accuracy of the cosmic X-ray background measurements is related to the intrinsic background variation in detectors $[3,4]$. This problem was comprehensively discussed at the Workshop on Electron Contamination in X-Ray Astronomy Experiments in 1974 [5]. It was shown that detectors of X-and gamma-ray on sounding rockets, on balloons, and on board the low-orbiting satellites are subject to in-orbit enhanced background noise caused by the magnetospheric electrons, especially in the Earth's auroral zone and zone of trapped radiation (radiation belt), that is, at high latitudes, and also in South Atlantic Anomaly (SAA) at low latitudes (see also [6,7]). To minimize the contamination, most cosmic and galactic $\mathrm{X}$ - and gamma-ray measurements made from within the magnetosphere are conducted at equatorial or low-to-middle magnetic latitudes, where the influence of auroral and radiation belt effects is expected to be small. It is considered that data below the radiation belt outside the region of SAA are particularly valuable to satellite missions in high-energy astrophysics. However, even then during the early 1970s, the X-ray astronomers unexpectedly revealed the electron-induced contaminant at relatively low latitudes as well, which was a few times higher than the cosmic Xray background $[2,8]$. They found several events when flux intensity of electrons with energy of tens of $\mathrm{keV}$ was as large as $\sim 10^{3} \mathrm{el} /\left(\mathrm{cm}^{2} \mathrm{~s} \mathrm{sr}\right)^{-1}$, exceeding quiet level by 2 orders of magnitude. Note that it is still much lower than in the radiation belt and auroral zones (see [9]). In addition to astrophysical measurements, ionospheric and atmospheric studies [10-17] and satellite data failures studies (e.g., $[18,19])$ also found several effects suggesting that electron impact is important factor at low and middle latitudes. That is, more importantly, the occasional electron flux increases below the radiation belt were discovered even earlier in direct satelliteborne measurements $[20,21]$ and then corroborated in several studies [22-25]. They reported about sporadic fluxes of very high intensity which was comparable with the auroral precipitation. However, the direct observations of sporadic events caused strong argument due to a doubt about validity of measured high intensity (see review by Paulikas [26]). 


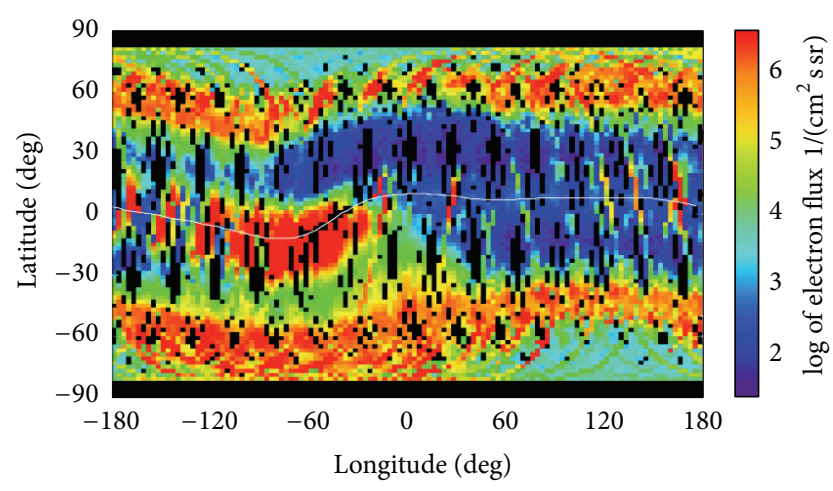

(a)

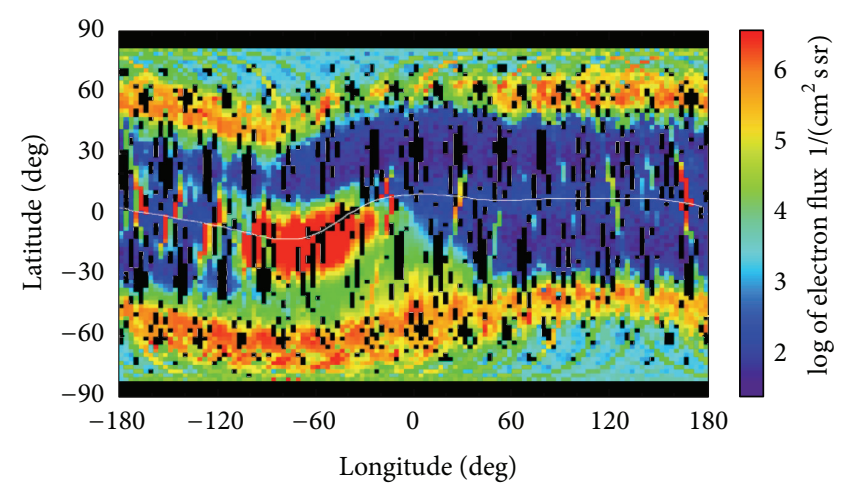

(b)

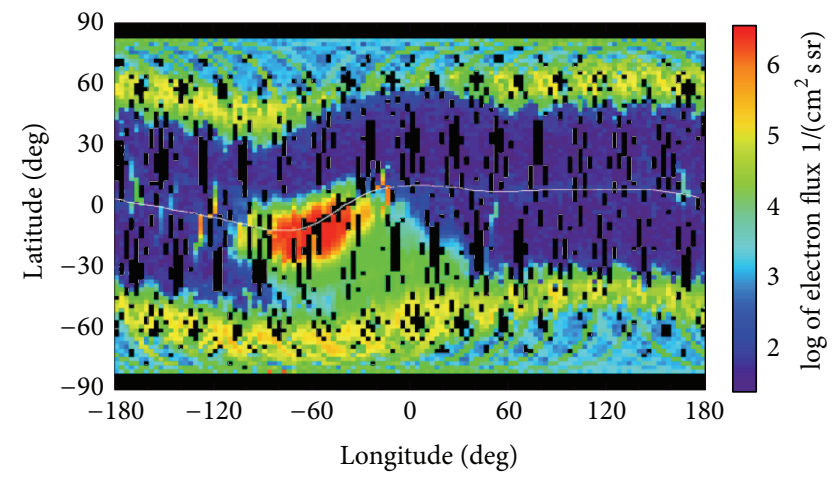

(c)

FIGURE 1: Energetic electron enhancement during the major geomagnetic storm on 15-16 May 2005. Global distribution of the electron fluxes in energy range: (a) $>30 \mathrm{keV}$; (b) $>100 \mathrm{keV;} \mathrm{(c)}>300 \mathrm{keV}$ measured by the NOAA/POES-15, $-16,-17$ satellites at altitude of $\sim 850 \mathrm{~km}$. The maps are composed of data retrieved from two orthogonally directed detectors (see details in the text). The white curve indicates the geomagnetic equator. Intensity of energetic electron fluxes extremely and globally enhances at equator-to-low latitudes (IRB and below it) even exceeding one at high latitudes (ORB and auroral zone).

As a result of this, despite importance of low-latitude measurement of electron fluxes recognized earlier [5], further investigation of the enhanced electrons phenomenon was not carried out.

Until recently, sporadic enhancement of energetic electrons below the inner radiation belt (IRB) was a poorstudied phenomenon [15, 27]. Comprehensive studies based on large statistics collected for more than ten years [28-31] have showed that fluxes of quasitrapped electrons within the energy range $10-300 \mathrm{keV}$ can increase dramatically by a few orders of magnitude relative to the quiet level at very low $L$ shells $(L<1.1)$, in a region called a forbidden zone. The most extreme intensity of forbidden zone fluxes of the order of auroral precipitation, $\sim 10^{6}-10^{7}\left(\mathrm{~cm}^{2} \mathrm{~s} \mathrm{sr}\right)^{-1}$, was observed during some major storms driven by a coronal mass ejection (CME). Nevertheless, CME-type or major storms themselves are not a necessary condition for electron enhancements in the forbidden zone. Another important solar wind driver resulting in significant flux enhancements is the extremely strong solar wind dynamic pressure, as it occurred on 21 January 2005 [32]. It can be easily understood that large enhancements occur much less frequently than moderate ones. The moderate fluxes are smaller by one-two orders of magnitudes. They are mostly associated with low-to-moderate level of geomagnetic activity and minor storms (major storms can also contribute, though). Minor storms, as known, are mainly driven by corotating interaction regions (CIR) and high speed solar wind streams (HSS) [33].

This paper describes the results of a statistical analysis of the energetic electron enhancements observed by a fleet of NOAA/POES low-orbiting satellites over the time period from 1999 to 2012. We demonstrate geographical distributions of great and moderate enhancements caused by different type of the solar wind drivers.

\section{Data from NOAA Satellites}

We used time profiles of $30-300 \mathrm{keV}$ electrons fluxes measured on board the polar orbiting NOAA/POES satellites [34]. The POES satellites have Sun-synchronous orbits at altitudes of $\sim 800-850 \mathrm{~km}$ (with $\sim 100$ minute periods of revolution). It is well known that the electron measurements can be distorted by proton contamination and nonideal detector efficiency. According to a comprehensive study [35] the $30 \mathrm{keV}$ electron fluxes should be, on an average, more than two times larger than uncorrected fluxes. The $100 \mathrm{keV}$ 


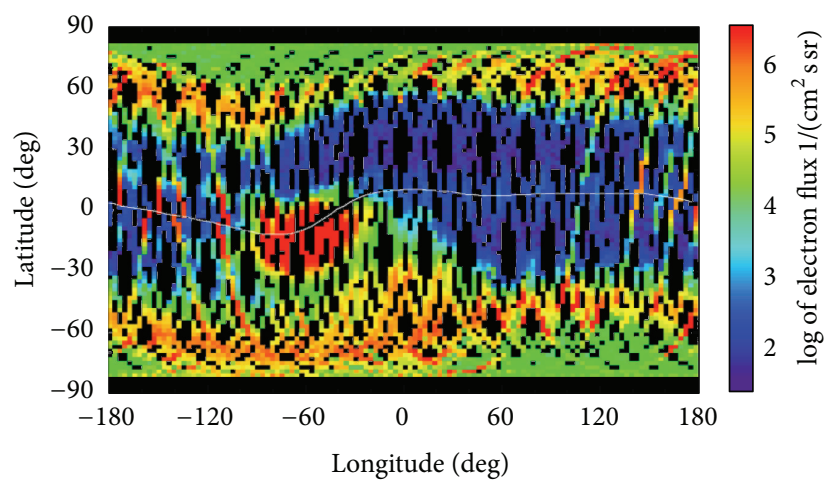

(a)

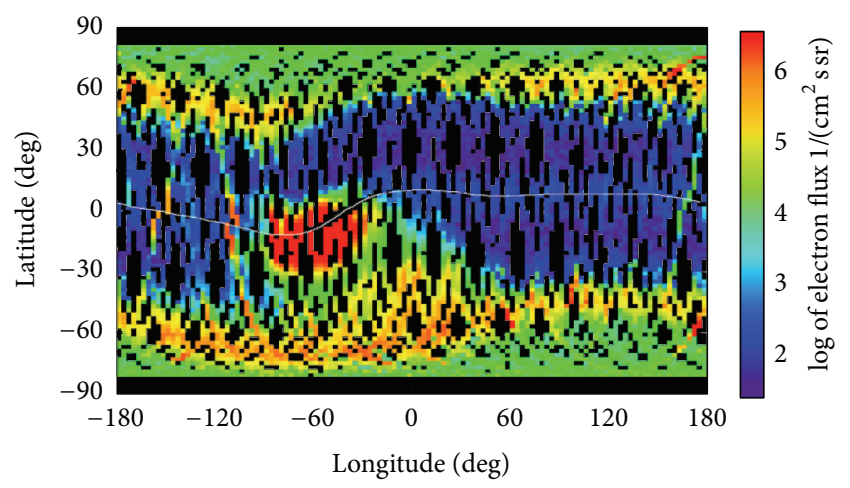

(b)

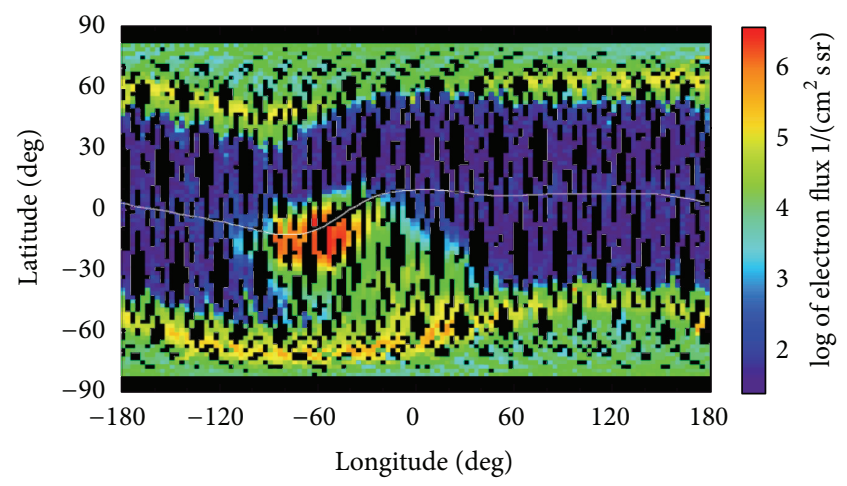

(c)

FIGURE 2: The same as Figure 1 but for extremely strong magnetospheric compression and moderate geomagnetic storm on 21 January 2005.

electron fluxes practically do not change, while the $300 \mathrm{keV}$ electron fluxes should be decreased by about twenty percent. Because this factor is not crucial for the current study, we present uncorrected fluxes.

\section{Enhancements of the Quasitrapped Energetic Electrons}

Figure 1 presents geographical distributions of energetic electron fluxes in three energy ranges: $>30 \mathrm{keV},>100 \mathrm{keV}$, and $>300 \mathrm{keV}$. The data was compiled from measurements by two orthogonally oriented detectors $\left(0^{\circ}\right.$-detector and $90^{\circ}$ detector) of three POES satellites (NOAA-15, NOAA-16, NOAA-17) during the major (CME-driven) geomagnetic storm on 15-16 May 2005. In each spatial bin, the maximal value of flux instead of the averaged one was used. The flux intensities below IRB in all three energy bands exceeded the quiet level by 5-6 orders of magnitude. The lower energy electrons outside the SAA region achieved an extremely large value of $3 \cdot 10^{7}\left(\mathrm{~cm}^{2} \mathrm{~s} \mathrm{sr}\right)^{-1}$, as much as in the IRB (including SAA) and auroral zone with outer radiation belt. The enhancements in $>30 \mathrm{keV}$ and $>100 \mathrm{keV}$ were long-lasting. The most prolonged equatorial enhancement in $>30 \mathrm{keV}$ occupied the forbidden zone at $L$ shell of 1.05-1.15 for more than 20 hours.
Figure 2 presents geographical distributions of energetic electron fluxes during the prolonged compression of the magnetosphere by extremely high solar wind dynamic pressure of more than $150 \mathrm{nPa}$ [32]. The magnetic storm was of moderate strength. Due to the compression, the Earth's magnetopause shrunk to about $\sim 3-4 \mathrm{Re}$ in the subsolar region, radiation belt, and ring current moved closely to the Earth. The enhancement of $>30 \mathrm{keV}$ electrons was observed during 6 hours. Fluxes of electrons with higher energies were also increased, but due to fast azimuthal drift they disappeared in one or two hours.

Figure 3 demonstrates that $>30 \mathrm{keV}$ electrons can appear even during weak geomagnetic storms. The global map was compiled from measurements by five NOAA/POES satellites for one year 2008. During this year of solar activity minimum, there were only minor (CIR/HSS-driven) storms of intensities less than $50 \mathrm{nT}$. In course of the year there were 60 days when the electron fluxes below IRB increased to $\sim 10^{4}$ $\left(\mathrm{cm}^{2} \mathrm{~s} \mathrm{sr}\right)^{-1}$. However, even moderate electron enhancements can significantly contaminate to the X-ray background.

\section{Summary}

In this paper we are concerned with a very important problem of the electron contamination to high-energy astrophysical measurements. The study is based on long-term statistics of 


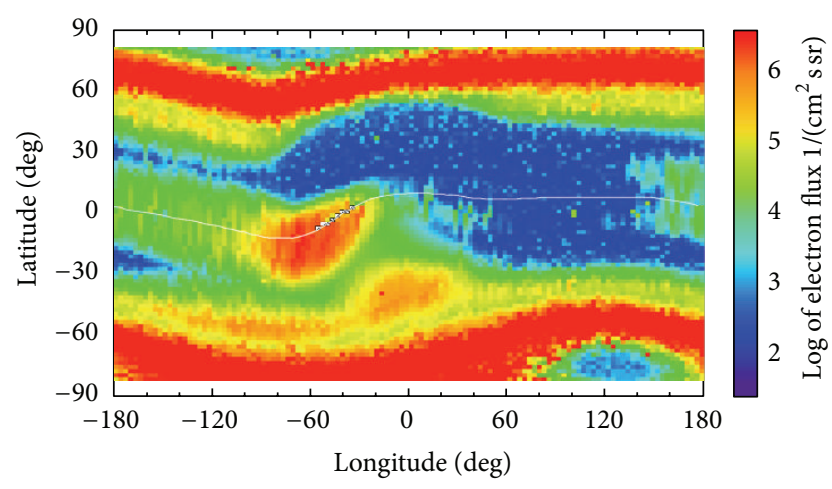

FIGURE 3: Global distribution of the electron fluxes in energy range $>30 \mathrm{keV}$ measured by the NOAA/POES-15, -16, -17, -18, and METOP-02 satellites during the whole year 2008 of the solar activity minimum. Low-latitude electron enhancements were observed during only 60 days in the course of the year.

the energetic electron observations by low-orbiting satellites. We have demonstrated three cases of electron fluxes that significantly exceeded a quite level: a major geomagnetic storm, a strong compression of the magnetosphere, and one-year period of the solar activity minimum leading to a weak geomagnetic activity.

The phenomenon of "forbidden zone electron" relates to the magnetospheric electric fields driven by external parameters, the solar wind, and interplanetary electric field [31]. A notable feature of the "forbidden-zone" $30 \mathrm{keV}$ electrons is their long persistence for about several hours. It is important that the significant and longtime electron enhancements at equatorial latitudes occur quite often during moderate CIR/HSS-storms.

\section{Conflict of Interests}

The authors declare that there is no conflict of interests regarding the publication of this paper.

\section{Acknowledgments}

The authors thank a team of NOAA's Polar Orbiting Environmental Satellites for providing experimental data about energetic particles. The work of Alla V. Suvorova was supported by Grant NSC-102-2811-M-008-045 from the National Science Council of Taiwan. Alla V. Suvorova and Alexei V. Dmitriev gratefully acknowledge the support of part of this work by Grant NSC103-2923-M-006-002-MY3/14-0592002HHC_a from Taiwan-Russia Research Cooperation.

\section{References}

[1] A. J. Dean, F. Lei, and P. J. Knight, "Background in space-borne low-energy $\gamma$-ray telescopes," Space Science Reviews, vol. 57, no. 1-2, pp. 109-186, 1991.

[2] F. D. Seward, R. J. Grader, A. Toor, G. A. Burginyon, and R. W. Hill, "Electrons at low altitudes: a difficult background problem for soft X-ray astronomy," in Proceedings of the Workshop on
Electron Contamination in X-Ray Astronomy Experiments, S. S. Holt, Ed., pp. 661-74, 1974, NASA GSFC Rep. X-661-74-130.

[3] D. E. Gruber, J. L. Matteson, L. E. Peterson, and G. V. Jung, “The spectrum of diffuse cosmic hard X-rays measured with HEAO 1," Astrophysical Journal Letters, vol. 520, no. 1, pp. 124-129, 1999.

[4] G. S. Bisnovatyi-Kogan and A. S. Pozanenko, "About the measurements of the hard X-ray background," Astrophysics and Space Science, vol. 332, no. 1, pp. 57-63, 2011.

[5] S. S. Holt, "Proceedings of the workshop on electron contamination in X-ray astronomy experiments, Washington, 26 April 1974," NASA GSFC Report X-661-74-130, 1974.

[6] R. Bučík, K. Kudela, A. V. Dmitriev, S. N. Kuznetsov, I. N. Myagkova, and S. P. Ryumin, "Spatial distribution of low energy gamma-rays associated with trapped particles," Advances in Space Research, vol. 30, no. 12, pp. 2843-2848, 2002.

[7] A. Gusev, I. Martin, and G. Pugacheva, "The soft X-ray emission of nocturnal atmosphere during the descending phase of the 23rd solar cycle," Sun and Geosphere, vol. 7, no. 2, pp. 127-131, 2012.

[8] J. E. Neighbours and G. W. Clark, "A survey of trapped low energy electrons near the inner boundary of the inner radiation zone from the OSO-7," in Proceedings of the Workshop on Electron Contamination in X-Ray Astronomy Experiments, S. S. Holt, Ed., 1974, NASA GSFC Rep. X-661-74-130.

[9] R. Bučík, K. Kudela, A. V. Dmitriev, S. N. Kuznetsov, I. N. Myagkova, and S. P. Ryumin, "Review of electron fluxes within the local drift loss cone: measurements on CORONAS-I," Advances in Space Research, vol. 36, no. 10, pp. 1979-1983, 2005.

[10] L. A. Antonova and G. S. Ivanov-Kholodny, "Corpuscular hypothesis for the ionization of the night ionosphere," Geomagnetizm I Aeronomiya, vol. 1, no. 2, pp. 164-173, 1961.

[11] L. A. Antonova and T. V. Kazarchevskaya, "Measurements of soft electron streams in the upper atmosphere at altitude to 500 km," in Space Research, Transactions of the All-Union Conference on Space Physics, G. A. Skuridin, Y. L. Al'pert, V. I. Krasovskiy, and V. V. Shvarev, Eds., Science Publishing House, Moscow, Russia, June 1965.

[12] W. C. Knudsen and G. W. Sharp, "F2-region electron concentration enhancement from inner radiation belt particles," Journal of Geophysical Research, vol. 73, no. 19, pp. 6275-6283, 1968.

[13] R. H. Doherty, "Observations suggesting particle precipitation at latitudes below 40N," Radio Science, vol. 6, no. 6, pp. 639-646, 1971.

[14] T. A. Potemra and T. J. Rosenberg, "VLF propagation disturbances and electron precipitation at mid-latitudes," Journal of Geophysical Research, vol. 78, no. 10, pp. 1572-1580, 1973.

[15] A. V. Dmitriev and H.-C. Yeh, "Storm-time ionization enhancements at the topside low-latitude ionosphere," Annales Geophysicae, vol. 26, no. 4, pp. 867-876, 2008.

[16] A. V. Dmitriev, H.-C. Yeh, M. I. Panasyuk et al., "Latitudinal profile of UV nightglow and electron precipitations," Planetary and Space Science, vol. 59, no. 8, pp. 733-740, 2011.

[17] P. Bobik, M. Putis, M. Bertaina et al., "UV night background estimation in South Atlantic anomaly," in Proceedings of the 33rd International Cosmic Ray Conference (ICRC '13), Rio de Janeiro, Brazil, July 2013.

[18] A. V. Dmitriev, I. I. Guilfanov, and M. I. Panasyuk, "Data failures in the "Riabina-2" experiment on MIR orbital station," Radiation Measurements, vol. 35, no. 5, pp. 499-504, 2002.

[19] J. F. Fennell, J. L. Roeder, and H. C. Koons, "Substorms and magnetic storms from the satellite charging perspective," COSPAR Colloquia Series, vol. 12, pp. 163-173, 2002. 
[20] V. I. Krasovskii, Y. M. Kushner, G. A. Bordovskii, G. F. Zakharov, and E. M. Svetlitskii, "The observation of corpuscles by means of the third artificial earth satellite," Planetary and Space Science, vol. 5, no. 3, pp. 248-249, 1961.

[21] I. A. Savenko, P. I. Shavrin, and N. F. Pisarenko, "Soft particle radiation at an altitude of $320 \mathrm{~km}$ in the latitudes near the equator," Iskusstvennye Sputniki Zemli, vol. 11, pp. 75-80, 1962 (Russian), Planetary and Space Science, vol. 11, pp. 431-436, 1963.

[22] W. J. Heikkila, "Soft particle fluxes near the equator," Journal of Geophysical Research, vol. 76, no. 4, pp. 1076-1078, 1971.

[23] J. D. Winningham, “"Low energy (10 eV to $10 \mathrm{keV})$ equatorial particle fluxes", Proceedings of the Workshop on Electron Contamination in X-Ray Astronomy Experiments, edited by S.S. Holt," NASA GSFC Report X-661-74-130, 1974.

[24] R. A. Goldberg, "Rocket observation of soft energetic particles at the magnetic equator," Journal of Geophysical Research, vol. 79, pp. 5299-5303, 1974.

[25] R. Lieu, J. Watermann, K. Wilhelm, J. J. Quenby, and W. I. Axford, "Observations of low-latitude electron precipitation," Journal of Geophysical Research, vol. 93, pp. 4131-4133, 1988.

[26] G. A. Paulikas, "Precipitation of particles at low and middle latitudes," Reviews of Geophysics and Space Physics, vol. 13, no. 5, pp. 709-734, 1975.

[27] A. V. Suvorova, L.-C. Tsai, and A. V. Dmitriev, "On relation between mid-latitude ionospheric ionization and quasi-trapped energetic electrons during 15 December 2006 magnetic storm," Planetary and Space Science, vol. 60, no. 1, pp. 363-369, 2012.

[28] A. V. Suvorova, L.-C. Tsai, and A. V. Dmitriev, "On magnetospheric source for positive ionospheric storms," Sun and Geosphere, vol. 7, no. 2, pp. 91-96, 2012.

[29] A. V. Suvorova, L.-C. Tsai, and A. V. Dmitriev, "TEC enhancement due to energetic electrons above Taiwan and the West Pacific," Terrestrial, Atmospheric and Oceanic Sciences, vol. 24, no. 2, pp. 213-224, 2013.

[30] A. V. Suvorova, A. V. Dmitriev, and L.-C. Tsai, "Evidence for near-equatorial deposition by energetic electrons in the ionospheric F-layer," in Proceedings of the International Conference "Modern Engineering and Technologies of the Future", A. Khnykin, Ed., pp. 68-82, Krasnoyarsk, Russia, February 2013.

[31] A. V. Suvorova, A. V. Dmitriev, L.-C. Tsai et al., "TEC evidence for near-equatorial energy deposition by $30 \mathrm{keV}$ electrons in the topside ionosphere," Journal of Geophysical Research A: Space Physics, vol. 118, no. 7, pp. 4672-4695, 2013.

[32] A. V. Dmitriev, A. V. Suvorova, J.-K. Chao et al., "Anomalous dynamics of the extremely compressed magnetosphere during 21 January 2005 magnetic storm," Journal of Geophysical Research, vol. 119, pp. 877-896, 2005.

[33] B. T. Tsurutani, W. D. Gonzalez, A. L. C. Gonzalez et al., "Corotating solar wind streams and recurrent geomagnetic activity: a review," Journal of Geophysical Research B: Solid Earth, vol. 111, no. 7, Article ID A07S01, 2006.

[34] D. S. Evans and M. S. Greer, "Polar orbiting environmental satellite space environment monitor: 2. Instrument descriptions and archive data documentation," Technical Memo version 1.4, NOAA Space Environ. Lab, Boulder, Colo, USA, 2004.

[35] T. Asikainen and K. Mursula, "Correcting the NOAA/MEPED energetic electron fluxes for detector efficiency and proton contamination," Journal of Geophysical Research A: Space Physics, vol. 118, no. 10, pp. 6500-6510, 2013. 

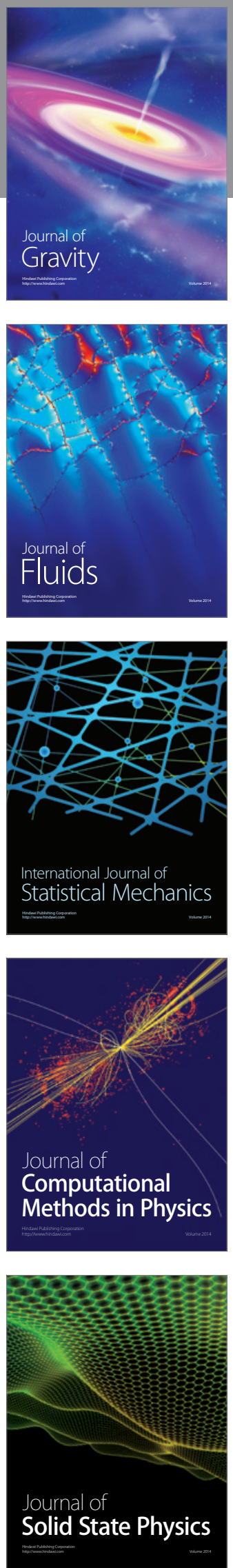

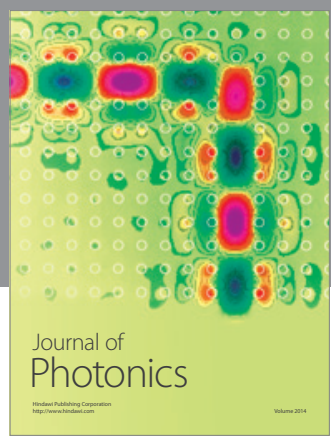

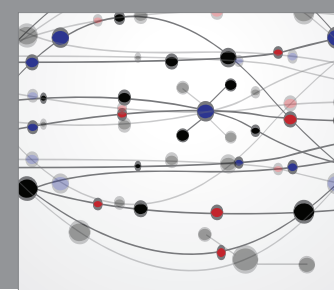

The Scientific World Journal

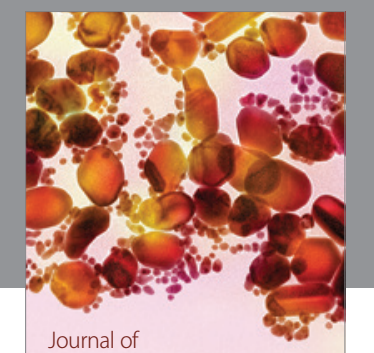

Soft Matter
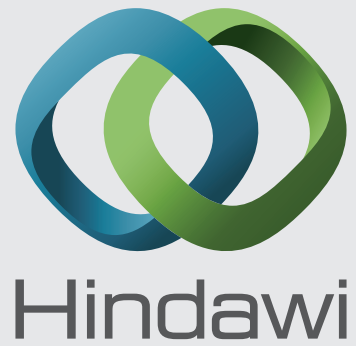

Submit your manuscripts at

http://www.hindawi.com
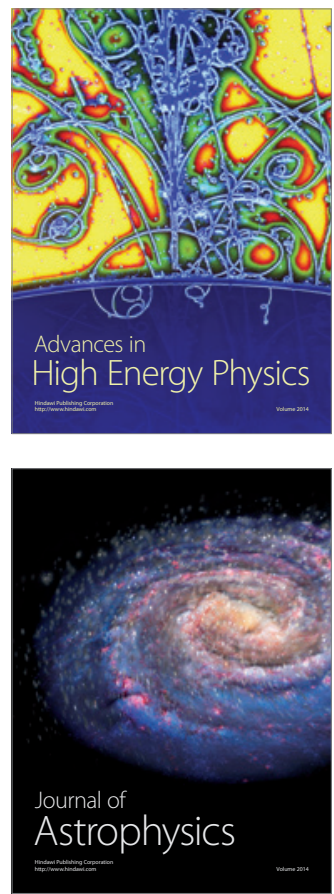
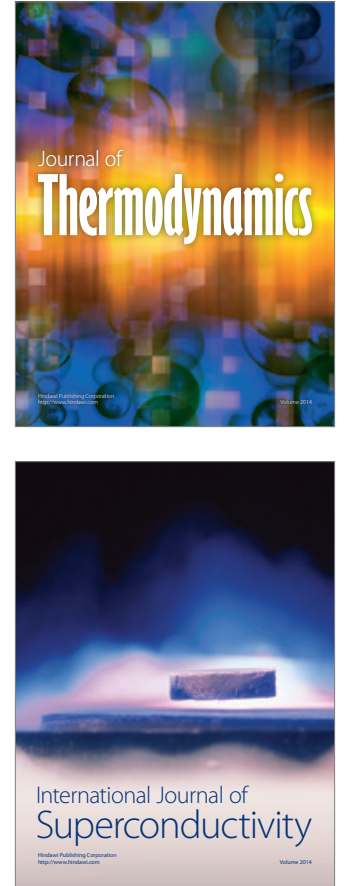
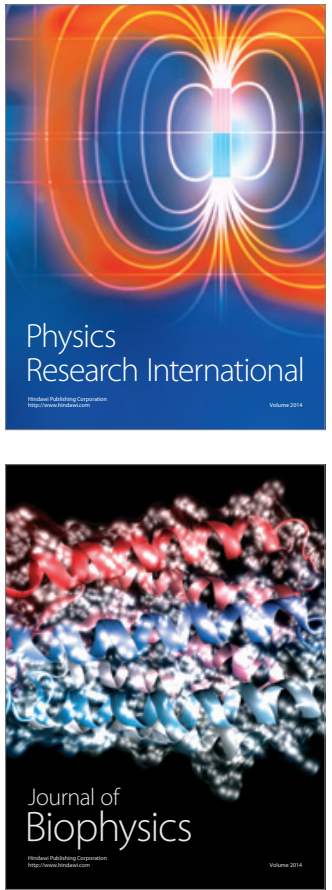
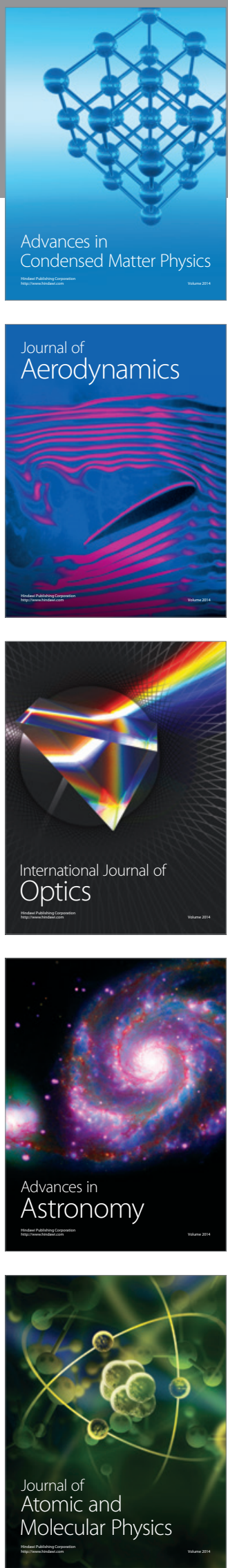